\section{Nota técnica}

Volumen 32(1):224-235. Enero-abril, 2021

e-ISSN 2215-3608, doi:10.15517/am.v32i1.41399

http://www.revistas.ucr.ac.cr/index.php/agromeso

\title{
Crecimiento de terneras F1 y F2 Kiwi Cross x Holstein ${ }^{1}$
}

\section{Growth of F1 and F2 calf Kiwi Cross x Holstein}

\author{
David Felipe Nieto-Sierra², Edmundo Andrés Timarán-Rivera ${ }^{3}$, José Carlos Montes-Vergara ${ }^{3}$, \\ Edwin Castro-Rincón $n^{3}$, Henry David Mogollón-García ${ }^{4}$
}

1 Recepción: 30 de abril, 2020. Aceptación: 31 de agosto, 2020. Este trabajo fue parte del proyecto "Modelo de producción láctea con tecnología de Nueva Zelanda adaptada a los trópicos colombianos" y fue financiado por la Corporación Colombiana de Investigación Agropecuaria (AGROSAVIA). Colombia.

2 Corporación Colombiana de Investigación Agropecuaria (AGROSAVIA). Centro de Investigación El Nus. Corregimiento de San José del Nus, San Roque, Antioquia, Colombia. dnieto@agrosavia.co (https://orcid.org/0000-0003-4808-1201).

3 Corporación Colombiana de Investigación Agropecuaria (AGROSAVIA). Centro de Investigación Obonuco. Km 5, Vía Pasto, Obonuco, Nariño, Colombia. ecastro@agrosavia.co (autor para la correspondencia; https://orcid.org/0000-0001-9841-8242), etimaran@agrosavia.co (https://orcid.org/0000-0002-8203-4081), jcmontes@agrosavia.co (https://orcid.org/0000-0001-8455-9060).

4 Universidad Estadual Paulista Júlio de Mesquita Filho, Facultad de Medicina Veterinaria y Zootecnia, Departamento de Radiología Animal y Reproducción Animal, Botucatu, Sao Paulo, Brazil. mogollongarcia@hotmail.com (https://orcid.org/0000-0003-0820-5847).

\section{Resumen}

Introducción. En el ciclo de producción de los bovinos de leche, el tiempo de crecimiento de las terneras representan un período de gastos y poco retorno para el productor, sin embargo, la preparación de hembras saludables durante este período es fundamental para incorporar al hato hembras productivas que garanticen el retorno financiero. Objetivo. Determinar los parámetros productivos y bovinométricos de las filiales uno (F1) y dos (F2) producto del cruce entre las razas de bovinos Kiwi Cross x Holstein. Materiales y métodos. Este trabajo se realizó en el Centro de Investigación Obonuco de la Corporación Colombiana de Investigación Agropecuaria (AGROSAVIA), ubicado en Pasto, Nariño, Colombia. Se utilizaron veinte hembras F1 (1/2 Kiwi Cross - 1/2 Holstein-Friesian) y veinte F2 (3/4 Kiwi Cross - 1/4 Holstein-Friesian). Se registraron y compararon variables productivas y bovinométricas durante el primer y sétimo mes de edad. Resultados. Las terneras F1 en el tercer mes presentaron mayor peso en comparación a las F2 $(158,9 \pm 2,5$ vs $115 \pm 2,5 \mathrm{~kg}$, respectivamente). Ambas generaciones presentaron una disminución de ganancia diaria de peso entre el tercer y sétimo mes de edad. En el sétimo mes las terneras F1 presentaron mayores valores en comparación a las F2 en las variables perímetro de tórax $(126,5 \pm 1,0 \mathrm{~cm}$ vs $116,9 \pm 0,9 \mathrm{~cm})$, altura a la cruz $(102,4 \pm 0,7$ $\mathrm{cm}$ vs $97,0 \pm 0,7 \mathrm{~cm})$, altura al anca $(107,3 \pm 0,7 \mathrm{vs} 101,2 \pm 0,7 \mathrm{~cm})$, distancia entre íleon e isquion $(34,5 \pm 0,4 \mathrm{vs} 32,3 \pm 0,4$ $\mathrm{cm})$ y distancia entre isquiones $(11,2 \pm 0,1$ vs $10,2 \pm 0,1 \mathrm{~cm})$. Conclusión. Entre el tercer y séptimo mes de edad se observaron las principales diferencias entre las dos filiales en la mayoría de las variables, a medida que se aumentó el porcentaje de Kiwi Cross, se obtuvieron animales de menor tamaño y peso.

Palabras claves: cruce, ganado para leche, ganancia diaria, medidas bovinométricas, peso. 


\begin{abstract}
Introduction. In the dairy cattle production cycle, the calf growth time represents a period of expenses and little return for the producer, however, the preparation of healthy females during this period is essential to incorporate productive females into the herd that guarantee the financial return. Objective. To determine the productive and bovinometric parameters of the subsidiaries one (F1) and two (F2) resulting from the cross between the Kiwi Cross $x$ Holstein breeds. Materials and methods. This work was carried out at the Obonuco Research Center of the Colombian Corporation for Agricultural Research (AGROSAVIA), located in Pasto, Nariño, Colombia. Twenty F1 (1/2 Kiwi Cross - 1/2 Holstein-Friesian) and twenty F2 (3/4 Kiwi Cross - 1/4 Holstein-Friesian) calves were used. Production and bovinometric variables were registered during the first and seventh month of age. Results. F1 calves in the third month showed greater weight compared to the F2 $(158.9 \pm 2.5 \mathrm{vs.} 115 \pm 2.5 \mathrm{~kg}$, respectively). Both generations showed a decrease in daily weight gain between the third and seventh month of age. In the seventh month, the F1 calves showed higher values compared to the $\mathrm{F} 2$ in the variables perimeter of the thorax $(126.5 \pm 1.0 \mathrm{~cm}$ vs $116.9 \pm 0.9 \mathrm{~cm})$, height at the cross $(102.4 \pm 0.7 \mathrm{~cm}$ vs $97.0 \pm 0.7 \mathrm{~cm})$, height at the haunch $(107.3 \pm 0.7$ vs $101.2 \pm 0.7 \mathrm{~cm})$, distance between ileum and ischium ( $34.5 \pm 0,4$ vs $32.3 \pm 0.4 \mathrm{~cm}$ ) and distance between sitting bones (11.2 \pm 0.1 vs. $10.2 \pm 0.1 \mathrm{~cm})$. Conclusion. Between the third and seventh month of age, the main differences between the two subsidiaries were observed in most of the variables, as the percentage of Kiwi Cross increased, animals of smaller size and weight were obtained.
\end{abstract}

Keywords: crossing, dairy cattle, daily gain, bovinometric measurements, weight.

\title{
Introducción
}

La producción de leche en Colombia se caracteriza por tener dos sistemas productivos, el especializado y el doble propósito; el sistema especializado es más intensivo y se encuentra en zonas frías del trópico alto, mientras que el sistema doble propósito es característico de zonas tropicales bajas, de pequeños y medianos productores (Holmann et al., 2004; 2006). Colombia cuenta con un inventario total de cabezas de ganado bovino de 27239767 , de las cuales, el 63,4 \% corresponden a hembras, de estas el 15,9\% son menores a un año (Departamento Administrativo Nacional de Estadística, 2020).

La producción lechera del departamento de Nariño es especializada de pequeña escala, cerca de un $40 \%$ de los predios poseen menos de cinco hectáreas y alrededor de un $90 \%$ tienen una producción diaria igual o menor a 100 litros (Mejía, 2012). Sin embargo, Nariño ocupa el quinto lugar en el territorio colombiano en cuanto a producción de leche, representando el 7,5\% de la producción diaria nacional (21 847085 litros) (Departamento Administrativo Nacional de Estadística, 2020).

Existe gran diversidad de razas de bovinos destinadas a la producción especializada de leche en todo el mundo, la que más predomina es Holstein-Friesian, seguida de otras como Jersey, Pardo Suizo, Ayrshire y doble propósito como la Normando (Carulla \& Ortega, 2016). En el trópico alto de Colombia y del departamento de Nariño también predomina la raza Holstein-Friesian, seguida de Normando, Pardo Suizo, Jersey y cruces de Holstein con las anteriores (Solarte et al., 2009). El cruce de Holstein con Jersey se emplea con el objetivo de combinar los mejores atributos de ambas razas y obtener animales medianos a pequeños adaptados a terreros de ladera (Rowarth, 2013). A partir de este cruce se generó en Nueva Zelanda la raza Kiwi Cross, la cual se caracteriza por ser animales rústicos y adaptables, con buena calidad composicional de la leche, de tamaño mediano, buena capacidad reproductiva, 
longevos y de buenos aplomos (Montgomerie, 2005; Rowarth, 2013); esta raza ha predominado en Nueva Zelanda los últimos años en un 47,2\% (LIC \& NZ Dairy, 2016).

El estrés al destete no solo depende de la transición de leche a pasto, también se puede ver influenciado por el manejo preparto de las madres y el momento del destete. Se ha indicado que las crías de madres que se mantienen en pastoreo antes del parto presentan menos estrés al destete, debido a que logran mayor ganancia diaria de peso e ingesta de materia seca comparado con las crías de las madres que se mantienen estabuladas y semi estabuladas (Black et al., 2017). Por otro lado, en un estudio se afirmó que el destete temprano o tardío también influye, ya que se encontró que crías destetadas a los 56 y 91 días de edad presentaron una ingesta similar de materia seca, pero la GDP fue menor en los animales destetados a los 56 días (McCoard et al., 2019), lo cual indicó que no pudieron compensar completamente con solo forraje la pérdida de energía y nutrientes del sustituto de leche que emplearon (Khan et al., 2016). Una adecuada alimentación en la transición del destete permite que el estrés disminuya y se logre un buen desarrollo corporal y del rumen. La alimentación de animales durante la transición al destete debe ser balanceada en el suministro de concentrado y forraje para garantizar un $\mathrm{pH}$ ruminal medio y que no se presente acidosis ruminal que puede generar trastornos en los animales (Kim et al., 2016).

Los productores de Nueva Zelanda realizan un seguimiento muy estricto al peso y ganancia diaria de peso para garantizar un óptimo desarrollo y crecimiento de las hembras de reemplazo de raza Kiwi Cross, establecen ganancias diarias de peso (GDP) ideales para los 0 a 3 meses de $0,70 \mathrm{~kg} \mathrm{~d}^{-1} \mathrm{y}^{\mathrm{de}} 3$ a 7 meses de $0,60 \mathrm{~kg} \mathrm{~d}^{-1}$ (Macdonald \& Roche, 2011).

Establecer una GDP ideal durante las fases iniciales, permite alcanzar un desarrollo corporal óptimo y, de esta manera, disminuir el tiempo improductivo de las hembras. Se ha demostrado que una GDP menor a $0,40 \mathrm{~kg} \mathrm{~d}^{-1}$ compromete el desarrollo del animal, sin embargo, cuando la GDP es superior a $0,80 \mathrm{~kg} \mathrm{~d}^{-1}$ los animales pueden presentar un desarrollo corporal acelerado, lo que da como resultado una posible conformación inadecuada de la ubre, la cual se caracteriza por generar deposiciones de grasa e inhibir la formación de células secretoras de leche. Estudios al respecto han señalado que se reduce el desarrollo de la ubre si la GDP es superior a $0,7 \mathrm{~kg} \mathrm{~d}^{-1}, 0,9 \mathrm{~kg}$ $\mathrm{d}^{-1}$ y $1,0 \mathrm{~kg} \mathrm{~d}^{-1}$ (Knight \& Sorensen, 2001; Mourits et al., 1999), mientras que autores como Van-Amburgh et al. (1998) y Zanton \& Heinrichs (2005), afirmaron que, para un buen desarrollo de la ubre, el límite superior de GDP debe ser entre 0,7 a $0,8 \mathrm{~kg} \mathrm{~d}^{-1}$.

Para llevar un control de estos indicadores productivos, se recomienda hacer un seguimiento mensual acompañado de la toma de medidas bovinométricas (medidas corporales) que son claves para determinar el crecimiento y tamaño corporal de los animales de reemplazo; medidas como el perímetro de tórax, altura de la cruz, altura del anca y ancho de la cadera se relacionan mejor con el tamaño real del animal y se asocian con la facilidad al parto y el rendimiento productivo de la primera lactancia (Boro et al., 2016; Hoffman, 1997).

Garantizar un óptimo crecimiento y desarrollo de las hembras de reemplazo, permite mantener o incrementar el volumen de leche en los sistemas de producción (Boro et al., 2016). A pesar de la importancia que tiene la cría de estos animales, en algunas ocasiones son descuidados por no representar un retorno económico inmediato, además de la concepción de generar costos adicionales por mano de obra y suplementos balanceados (Boro et al., 2016; Hoffman, 2015).

Dado que en el centro de investigación Obonuco se encuentra en validación el uso de cruzamientos entre animales Kiwi Cross x Holstein, se hace necesario determinar las características fenotípicas de sus filiales. Las medidas bovinométricas son una opción para complementar la selección de los animales de reemplazo, por lo cual este estudio tuvo como propósito determinar los parámetros productivos y bovinométricos de las filiales uno (F1) y dos (F2) producto del cruce entre las razas Kiwi Cross x Holstein. 


\section{Materiales y métodos}

\section{Localización}

El experimento se realizó entre junio de 2015 y junio de 2017 en las instalaciones del Centro de Investigación Obonuco de la Corporación Colombiana de Investigación Agropecuaria (AGROSAVIA), ubicado en Pasto, Nariño, Colombia; a una altura de $2858 \mathrm{msnm}$, temperatura promedio de $13{ }^{\circ} \mathrm{C}$ y precipitación promedio anual de $800 \mathrm{~mm}$ (Mogollón et al., 2020).

\section{Animales}

Se emplearon veinte hembras F1 (1/2 Kiwi Cross - 1/2 Holstein-Friesian) y veinte hembras F2 (3/4 Kiwi Cross - 1/4 Holstein-Friesian) clínicamente saludables, con edad de siete meses. Posterior al nacimiento, toda ternera se observó para un correcto amamantamiento y se realizó la desinfección de ombligo durante los tres días siguientes. Al tercer día del nacimiento, las terneras se apartaron de la madre y se alimentaron individualmente con 51 de leche al día hasta los tres meses de edad, dividido en dos tomas (06:00 y 15:00 hrs), luego del segundo suministro de leche (15:00 hrs) se resguardaron todas las noches en grupo en un corral de cemento $15 \mathrm{x} 5 \mathrm{~m}$. Las terneras se suplementaron en grupo, entre el mes 1 y 3 con $300 \mathrm{~g} \mathrm{día}^{-1}$ (proteína $18 \%$, grasa 2,5\%, fibra $12 \%$, cenizas $10 \%$, humedad, $13 \%$ ) y de 3 a 7 meses con $500 \mathrm{~g} \mathrm{día}^{-1}$ (proteína $16 \%$, grasa $2,5 \%$, fibra $15 \%$, cenizas $10 \%$, humedad, $13 \%$ ). El consumo de agua fue ad libitum y se mantuvieron en potreros con mezclas de gramíneas y leguminosas como Raygrass de las variedades Samson, Sterling, Ohao y One 50 (Lolium multiflorum), trébol (Trifolium repens), Saboya (Holcus lanatus), Azul Orchoro (Dactylis glomerata) y Kikuyo (Cenchrus clandestinum). Esta mezcla de gramíneas y leguminosas presentó una composición nutricional en materia seca de $22 \%$, proteína cruda de $19 \%$, fibra en detergente neutro de $49 \%$, fibra en detergente ácido de $24 \%$, lignina de $6 \%$, hemicelulosa de $25 \%$ y digestibilidad de $67 \%$. Se estimó un consumo de materia seca del $3 \%$. Las desparasitaciones internas se realizaron con productos a base de Fenbendazol sumado a la aplicación de complejos multivitamínicos, con una frecuencia de cada tres meses.

\section{Variables medidas}

Las variables se registraron mensualmente, desde el primer mes de edad hasta el mes siete. Los animales se trasladaron al corral en horas de la mañana (08:00 hrs) y se ingresaron al embudo para realizar las medidas bovinométricas, para evitar el movimiento y asegurar una correcta posición. Luego de medirlas, todas las terneras se pesaron $\mathrm{y}$, posteriormente, se regresaron al potrero.

\section{Variables productivas}

Las variables productivas consideradas fueron el peso $(\mathrm{kg})$ y la ganancia diaria de peso (GDP), las cuales se midieron desde el primer mes hasta el mes siete de edad. Para tal fin, se empleó la báscula digital (GB5 ROJO TEK ${ }^{\circledR}$ ).

\section{Variables bovinométricas}

Las medidas bovinométricas se registraron en centímetros $(\mathrm{cm})$ y se tomaron con una regla y cinta métrica, para luego discriminarlas por plano anatómico. Se evaluaron en miembro anterior: perímetro de tórax (PT) y la 
altura a la cruz (AC); y miembro posterior: altura al anca (AA), distancia entre íleon e isquion (DII) y distancia entre isquiones (DI).

Las medidas se registraron de la siguiente manera: PT: medida del perímetro alrededor del pecho, pasando por la cruz y el esternón; AC: medida de la distancia del suelo al punto más elevado de la cruz; AA: medida de la distancia del suelo al punto más elevado de la grupa; DII: medida desde la punta de la cadera (ilion) hasta el isquion; DI: distancia entre la punta de los isquiones (World Holstein-Friesian Federation [WHFF], 2005; Martínez et al., 2007; de-Escobar et al., 2014). Al igual que el peso, todas las medidas bovinométricas se tomaron cada mes.

\section{Análisis estadístico}

Presupuestos de normalidad y homogeneidad se determinaron con base en la prueba de Shapiro Wilk y Barlett, respectivamente. Se utilizó un modelo linear mixto (Proc Mixed). Las matrices de covarianza se seleccionaron con base en los modelos AIC (Akaike's Information Criterion), AICC (Consistent Akaike's Information Criterion) y BIC (Bayesian Information Criterion), la comparación entre las medias se realizó con el empleo de pdiff. Se utilizaron como efectos fijos el cruce (C) y la edad (E), así como su interacción (C*E). Los resultados se muestran como media \pm error estándar (EE), diferencias significativas se consideraron cuando $\mathrm{p}<0,05$. El paquete estadístico utilizado fue SAS versión 9.4 (SAS Institute Inc., Cary, NC, USA).

\section{Resultados}

\section{Variables productivas}

El peso y la ganancia diaria de peso (GDP) (Figura 1A y 1B) mostraron efecto del cruce y la edad $(\mathrm{p}<0,0001) \mathrm{e}$ interacción entre el cruce y la edad ( $\mathrm{C} * \mathrm{E} \mathrm{p}<0,0001$ y $\mathrm{p}<0,0008$, respectivamente). Se observó diferencia estadística ( $<<0,05)$ entre F1 y F2 en los meses cuatro $(109,1 \pm 2,6$ vs 94,3 $\pm 3,1 \mathrm{~kg})$, cinco $(129,2 \pm 2,6$ vs $94,6 \pm 2,9 \mathrm{~kg})$, seis $(147,3 \pm 2,6$ vs $112,8 \pm 2,9 \mathrm{~kg})$ y siete $(158,9 \pm 2,6$ vs $115,0 \pm 2,5 \mathrm{~kg})$, con diferencia entre las dos filiales de $14,8 \pm 0,5$ $\mathrm{kg}, 34,5 \pm 0,3 \mathrm{~kg}, 34,5 \pm 0,3 \mathrm{~kg}$ y $43,9 \pm 0,1 \mathrm{~kg}$, respectivamente. Se exhibió una mayor diferencia entre los meses cinco, seis y siete (Figura 1A).

La ganancia diaria de peso (GDP) fue mayor en la F1, presentó diferencia $(\mathrm{p}<0,05)$ en los meses cuatro $\left(0,72 \pm 0,05\right.$ vs $\left.0,48 \pm 0,07 \mathrm{~kg} \mathrm{~d}^{-1}\right)$, cinco $\left(0,64 \pm 0,05\right.$ ss $\left.0,12 \pm 0,06 \mathrm{~kg} \mathrm{~d}^{-1}\right)$, seis $\left(0,57 \pm 0,05\right.$ vs $\left.0,26 \pm 0,06 \mathrm{~kg} \mathrm{~d}^{-1}\right)$ y siete $\left(0,38 \pm 0,05\right.$ vs $\left.0,17 \pm 0,05 \mathrm{~kg} \mathrm{~d}^{-1}\right)$, con diferencia entre las dos filiales de $0,24 \pm 0,01 \mathrm{~kg} \mathrm{~d}^{-1}, 0,51 \pm 0,01 \mathrm{~kg} \mathrm{~d}^{-1}, 0,32 \pm 0,01$ $\mathrm{kg} \mathrm{d}^{-1}$ y $0,21 \pm 0,01 \mathrm{~kg} \mathrm{~d}^{-1}$, respectivamente. A partir del mes tres se observó un patrón decreciente en la GDP para las dos filiales, la mayor diferencia se presentó en los meses cinco y seis (Figura 1B).

\section{Variables bovinométricas}

Se observó (Figura 2A y 2B) efecto del cruce y la edad para perímetro de tórax (PT) y altura a la cruz (AC) $(\mathrm{p}<0,0001)$ e interacción entre el cruce y la edad ( $\mathrm{C} * \mathrm{E} \mathrm{p}<0,0021$ y $\mathrm{p}<0,0001$, respectivamente). Durante los siete meses la F1 tuvo mayor PT en comparación a la F2, con diferencia estadística $(\mathrm{p}<0,05)$ en los meses uno $(83,0 \pm 1,0$ vs $78,8 \pm 1,1 \mathrm{~cm})$, cuatro $(110,8 \pm 1,0$ vs $106,3 \pm 1,2 \mathrm{~cm})$, cinco $(116,5 \pm 1,0$ vs $109,6 \pm 1,1 \mathrm{~cm})$, seis $(122,7 \pm 1,0$ vs 115,4 $\mathrm{cm})$ y siete $(126,6 \pm 1,0$ vs $116,9 \pm 0,9 \mathrm{~cm})$, con diferencia de PT entre las dos filiales de 4,2 $\pm 0,1 \mathrm{~cm}, 4,5 \pm 0,2 \mathrm{~cm}$, $6,9 \pm 0,1,7,2 \pm 0,1 \mathrm{~cm}$ y $9,7 \pm 0,1 \mathrm{~cm}$, respectivamente. La mayor diferencia se observó entre los meses cinco y siete (Figura 2A). 

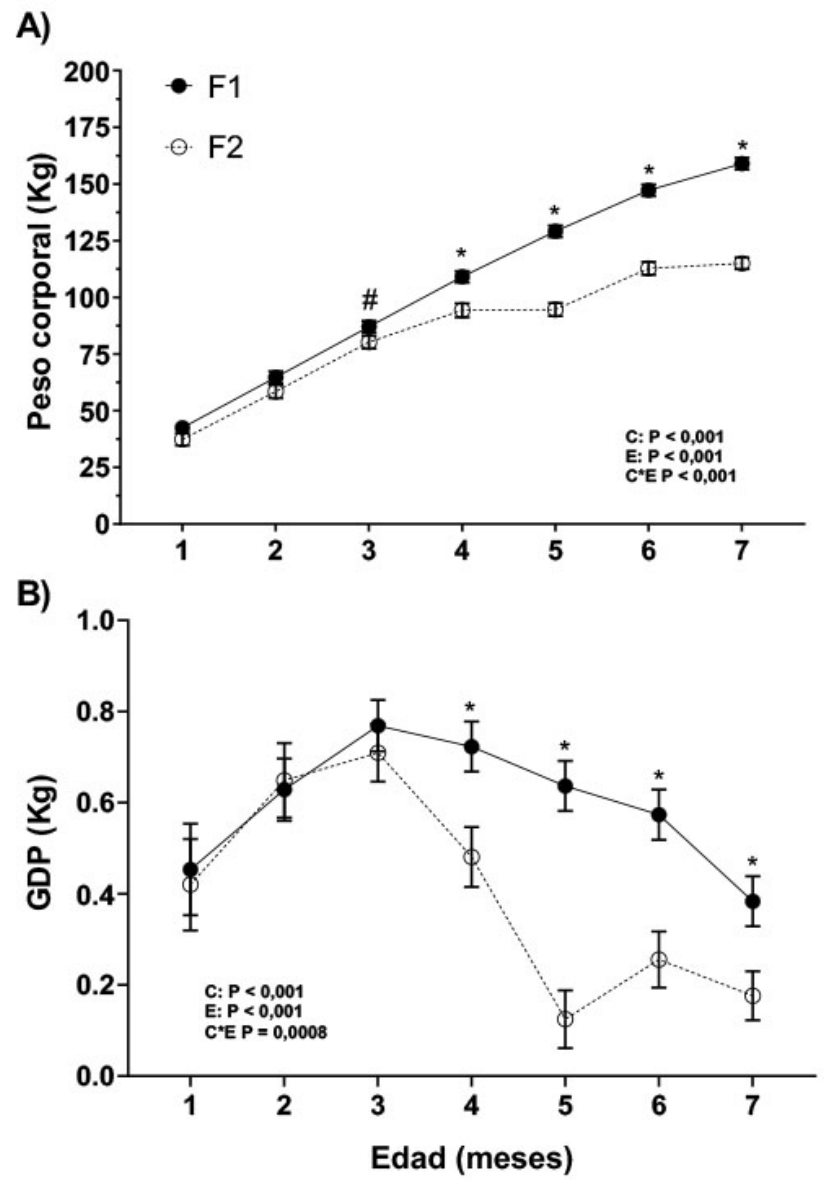

Figura 1. Promedio \pm EE de peso (A) y ganancia diaria de peso (GDP) (B) de la F1 (1/2 Kiwi Cross - 1/2 Holstein-Friesian) y F2 (3/4 Kiwi Cross - 1/4 Holstein-Friesian). Centro de Investigación Obonuco de la Corporación Colombiana de Investigación Agropecuaria (AGROSAVIA), Nariño, Colombia, 2017.

Probabilidades para los efectos de $(\mathrm{C}$, cruce; E, edad) y la interacción entre C*E son mostrados. (*) Indica diferencia estadística significativa $(\mathrm{p}<0,05)$ entre las filiales. (\#) Indica tendencia entre las filiales.

Figure. 1. Mean \pm SEM of weight (A) and daily weight gain (GDP) (B) of F1 (1/2 Kiwi Cross - 1/2 Holstein-Friesian) and F2 (3/4 Kiwi Cross - 1/4 Holstein-Friesian). Obonuco Research Center, Corporación Colombiana de Investigación Agropecuaria (AGROSAVIA), Nariño, Colombia, 2017.

Probabilities for the effects of $(\mathrm{C}$, crossover, $\mathrm{E}$, age) and the interaction between $\mathrm{C} * \mathrm{E}$ are shown. (*) Indicates significant statistical difference $(\mathrm{p}<0.05)$ between the subsidiaries. $\#)$ Indicates trend among subsidiaries.

La altura a la cruz (AC) fue mayor en la F1 con respecto a la F2 $(\mathrm{p}<0,05)$ en los meses cinco $(96,1 \pm 0,8 \mathrm{vs}$ $92,1 \pm 0,9 \mathrm{~cm})$, seis $(100,3 \pm 0,8$ vs $95,3 \pm 0,9 \mathrm{~cm})$ y siete $(102,4 \pm 0,7$ vs $97,0 \pm 0,7 \mathrm{~cm})$, con diferencia de AC entre las dos filiales de $3,9 \pm 0,1 \mathrm{~cm}, 4,9 \pm 0,1 \mathrm{~cm}$ y $5,5 \pm 0,1 \mathrm{~cm}$, respectivamente (Figura $2 \mathrm{~B}$ ).

La altura al anca (AA) y la distancia entre íleon e isquion (DII) (Figura 3A y 3B) presentaron efecto del cruce y la edad ( $\mathrm{p}<0,0001)$ e interacción entre el cruce y la edad ( $\mathrm{C} * \mathrm{E} \mathrm{p}<0,0071$ y $\mathrm{p}<0,007$, respectivamente). Asimismo, la 


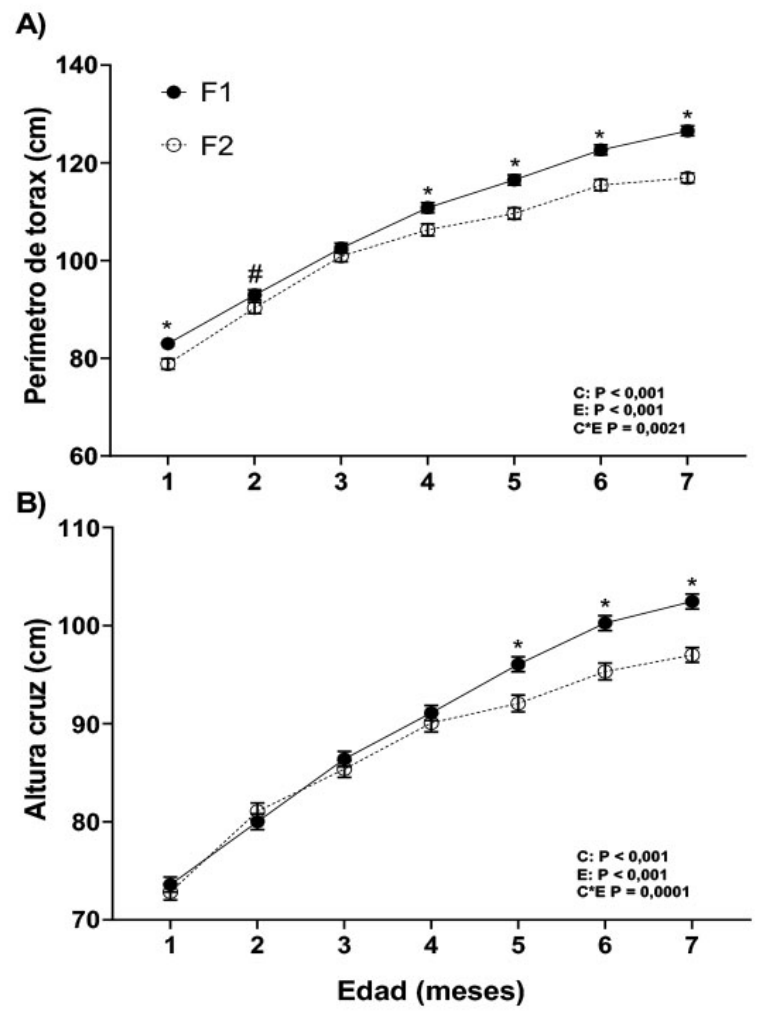

Figura 2. Promedio \pm EE de perímetro de tórax (PT) (A) y altura a la cruz (AC) (B) de la F1 (1/2 Kiwi Cross - 1/2 Holstein-Friesian) y F2 (3/4 Kiwi Cross - 1/4 Holstein-Friesian). Centro de Investigación Obonuco de la Corporación Colombiana de Investigación Agropecuaria (AGROSAVIA), Nariño, Colombia, 2017.

Probabilidades para los efectos de $(\mathrm{C}$, cruce; $\mathrm{E}$, edad) y la interacción entre $\mathrm{C} * \mathrm{E}$ son mostrados. (*) Indica diferencia estadística significativa $(\mathrm{p}<0,05)$ entre las filiales. (\#) Indica tendencia entre las filiales.

Figure 2. Mean \pm SEM of thorax perimeter (PT) (A) and withers height (AC)) (B) of F1 (1/2 Kiwi Cross - 1/2 Holstein-Friesian) and F2 (3/4 Kiwi Cross - 1/4 Holstein-Friesian). Obonuco Research Center, Corporación Colombiana de Investigación Agropecuaria (AGROSAVIA), Nariño, Colombia, 2017.

Probabilities for the effects of $\left(\mathrm{C}\right.$, crossover; E, age) and the interaction between $\mathrm{C}^{*} \mathrm{E}$ are shown. $\left(^{*}\right)$ Indicates significant statistical difference $(\mathrm{p}<0.05)$ between the subsidiaries. (\#) Indicates trend among subsidiaries.

distancia entre isquiones (DI) (Figura 3C) mostró efecto del cruce $(\mathrm{p}<0,0266)$ y la edad $(\mathrm{p}<0,0001)$ con interacción entre el cruce y la edad $(\mathrm{C} * \mathrm{E} \mathrm{p}<0,0266)$. La F1 presentó mayor AA $(\mathrm{p}<0,05)$ en comparación a la F2 en los meses uno $(80,4 \pm 0,8$ vs $77,3 \pm 0,8 \mathrm{~cm})$, tres $(92,6 \pm 0,7$ vs $90,3 \pm 0,8 \mathrm{~cm})$, cuatro $(97,4 \pm 0,8$ vs $95,0 \pm 0,9 \mathrm{~cm})$, cinco $(102,3 \pm 0,8$ vs $95,9 \pm 0,8 \mathrm{~cm})$, seis $(105,1 \pm 0,8$ vs $99,7 \pm 0,8 \mathrm{~cm})$ y siete $(107,3 \pm 0,7 v s 101,2 \pm 0,7 \mathrm{~cm})$ (Figura 3A).

La F1 presentó mayor DII y DI ( $\mathrm{p}<0,05)$ en comparación a la F2 en los meses cinco $(32,4 \pm 0,4 v s 29,9 \pm 0,5 \mathrm{~cm}$ y $10,5 \pm 0,2$ ss $9,8 \pm 0,2 \mathrm{~cm})$, seis $(33,7 \pm 0,4 v s 31,9 \pm 0,5 \mathrm{~cm}$ y $10,9 \pm 0,2 v s 10,4 \pm 0,2 \mathrm{~cm})$ y siete $(34,5 \pm 0,4 v s 32,3 \pm 0,4$ $\mathrm{cm}$ y $11,2 \pm 0,1$ vs $10,2 \pm 0,1 \mathrm{~cm}$ ) (Figura $3 \mathrm{~B}$ y $3 \mathrm{C}$ ). 


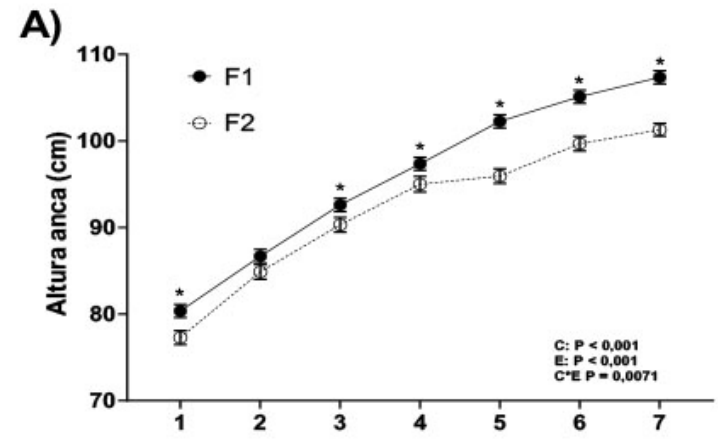

B)
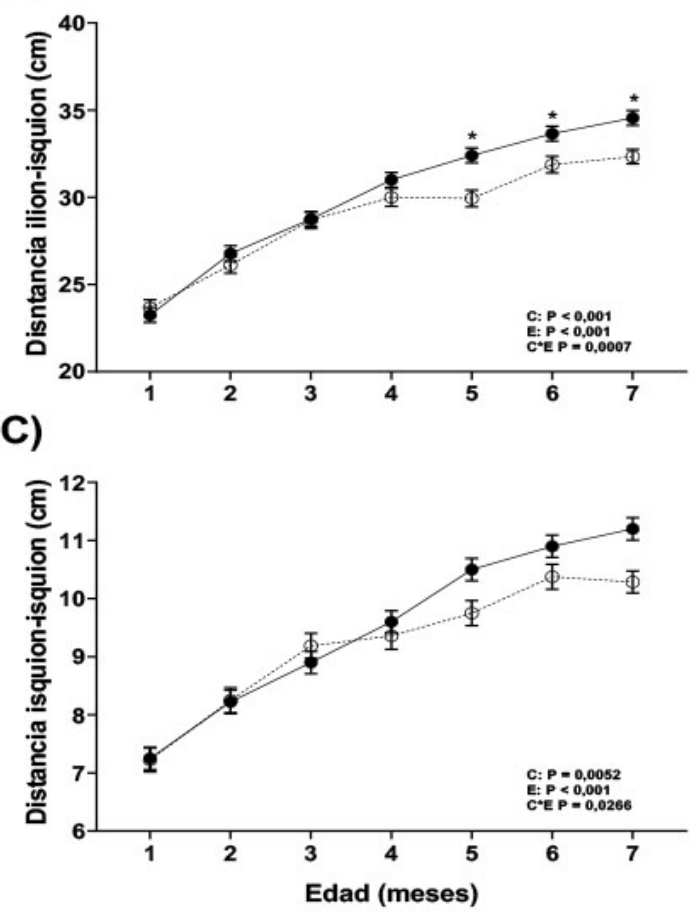

Figura 3. Promedio \pm EE de altura al anca AA (A), distancia entre íleon e isquion (DII) (B) y distancia entre isquiones (DI) (C) de la F1 (1/2 Kiwi Cross - 1/2 Holstein-Friesian) y F2 (3/4 Kiwi Cross - 1/4 Holstein-Friesian). Centro de Investigación Obonuco, Corporación Colombiana de Investigación Agropecuaria (AGROSAVIA), Nariño, Colombia, 2017.

Probabilidades para los efectos de (C, cruce; E, edad) y la interacción entre C*E son mostrados. (*) Indica diferencia estadística significativa $(\mathrm{p}<0,05)$ entre las filiales.

Figure 3. Mean \pm SEM of hip height (AA) (A), distance between ilio and ischium (DII) (B) and distance between ischial (DI) (C) of F1 (1/2 Kiwi Cross - 1/2 Holstein-Friesian) and F2 (3/4 Kiwi Cross - 1/4 Holstein-Friesian). Obonuco Research Center, Corporación Colombiana de Investigación Agropecuaria (AGROSAVIA), Nariño, Colombia, 2017.

Probabilities for the effects of $(\mathrm{C}$, crossover; $\mathrm{E}$, age) and the interaction between $\mathrm{C} * \mathrm{E}$ are shown. (*) Indicates significant statistical difference $(\mathrm{p}<0.05)$ between the subsidiaries. (\#) Indicates trend among subsidiaries. 


\section{Discusión}

Los pesos de la F1 y F2 en el tercer mes fueron inferiores a los reportados para la raza Holstein-Friesian $(98,2 \pm 4,2 \mathrm{~kg}$ ) (Bar-Peled et al., 1997), pero superiores a la raza Jersey $(72,5 \mathrm{~kg})$ (Monge-Rojas \& Elizondo-Salazar, 2017). Estos resultados pueden explicarse por el cruce absorbente F1 (1/2 Kiwi Cross - 1/2 Holstein-Friesian) y F2 (3/4 Kiwi Cross - 1/4 Holstein-Friesian), semejándose a la raza Jersey. Lo anterior coincide con Montgomerie (2005) y Rowarth (2013), quienes afirmaron que la raza Kiwi Cross se caracteriza por ser de tamaño mediano.

La ganancia diaria de peso (GDP) de la F1 y F2 en el tercer mes fue inferior a los valores reportados para la raza Jersey de $0,81 \mathrm{~kg} \mathrm{~d}^{-1}$ (Monge-Rojas \& Elizondo-Salazar, 2017) y de $0,83 \mathrm{~kg} \mathrm{~d}^{-1}$ para la raza Holstein-Friesian (Vargas-Ramírez \& Elizondo-Salazar, 2014); pero similar a la GDP obtenida por Bar-Peled et al. (1997) para la raza Holstein-Friesian $\left(0,71 \pm 0,10 \mathrm{~kg} \mathrm{~d}^{-1}\right)$. Entre ambas filiales, la diferencia fue marcada en GDP a partir del cuarto mes, tiempo para el cual fue notorio el efecto en el cambio de alimentación. Las hembras F1 se vieron menos afectadas en la transición de leche a pasto, adicionalmente, demostraron una ganancia de peso más constante y una disminución de la GDP menos drástica. Por lo tanto, se puede inferir que el estrés al destete fue menor en hembras con mayor proporción de raza Holstein.

Tanto el peso como la GDP permiten llevar un seguimiento del crecimiento de los animales, adicionalmente, se puede observar si las condiciones de alimentación y manejo necesarias para lograr su óptimo desarrollo y tomar las decisiones pertinentes de acuerdo con el caso. Estos dos parámetros son importantes en el logro de la pubertad de las hembras, ya que la madurez sexual está más influenciada por el peso (Boro et al., 2016; Wattiaux, 2011), la cual se alcanza cuando la hembra obtiene el 60 o $65 \%$ del peso adulto. Para lograr este peso objetivo, se deben asegurar ganancias diarias entre $0,50 \mathrm{~kg} \mathrm{~d}^{-1}$ (Boro et al., 2016) y $0,70 \mathrm{~kg} \mathrm{~d}^{-1}$ (Shamay et al., 2005). A pesar de que las filiales presentaron una reducción de peso y GDP luego de los tres meses (destete), las hembras se desarrollaron bien y alcanzaron su primer servicio con una edad y peso promedio de 17 meses y $280 \mathrm{~kg}$, respectivamente.

En Nueva Zelanda, para la raza Kiwi Cross establecieron GDP ideales para la fase de cría ( 0 a 3 meses) y levante (3 a 12 meses) de 0,70 y 0,60 kg d ${ }^{-1}$, respectivamente (Macdonald \& Roche, 2011). Con relación al presente estudio, los valores obtenidos entre los 0 y 3 meses para las dos filiales se encuentran en estos rangos, sin embargo, entre los 3 y 7 meses para la F1 y F2 estos valores fueron menores $\left(0,38 \pm 0,05 \mathrm{~kg} \mathrm{~d}^{-1}\right.$ vs $0,17 \pm 0,05 \mathrm{~kg} \mathrm{~d}^{-1}$, respectivamente) debido al destete. Es necesario determinar si en condiciones de trópico alto colombiano, el uso de las pasturas entre los tres y siete meses es menos eficiente o si otro factor posterior a la transición de leche a pasto está afectando la GDP. No obstante, datos reportados por Boro et al. (2016), coincidieron con las GDP obtenidas en el presente estudio entre los tres y siete meses.

Durante los siete meses de evaluación, las hembras F1 mostraron un perímetro de tórax (PT) mayor que las hembras F2, diferencia más maracada a partir del cuarto mes de edad. Los datos sugieren que hembras F1 tendrán una mayor talla que las F2. Al compararse las dos filiales en el tercer mes con animales mestizos con Holstein, presentaron un PT inferior (120,1 cm) (Kienitz et al., 2017). En un estudio, al sexto mes se obtuvo un PT de 128 $\mathrm{cm}$ y $114 \mathrm{~cm}$ para hembras Holstein-Friesian y Jersey, respectivamente (Macdonald et al., 2005), valores que fueron similares a los obtenidos para las filiales F1 y F2 del presente estudio.

Entre filiales, la altura a la cruz (AC) fue mayor en hembras F1 a partir del quinto mes. En el sexto mes los valores fueron similares a los reportados por Macdonald et al. (2005) para animales Holstein $(100,25 \pm 0,76 \mathrm{~cm})$ y Jersey $(95,31 \pm 0,85 \mathrm{~cm})$ en relación a las hembras F1 $(101 \mathrm{~cm})$ y F2 $(93 \mathrm{~cm})$ del presente estudio, respectivamente. Este parámetro se considera importante porque los animales altos tienen cuerpos más largos, y por lo tanto, tienen más capacidad corporal, además de mantener la ubre alejada del suelo, lo cual disminuye lesiones y facilita las operaciones del ordeño (Boro et al., 2016). 
En el miembro posterior, las medidas de altura al anca (AA), distancia entre íleon e isqueon (DII) y distancia entre isquiones (DI) presentaron diferencia durante el tercer y séptimo mes, los valores de la F1 fueron mayores en comparación con la F2; similar a un estudio que reportó una mayor AA al tercer y cuarto mes de edad en animales mestizos con Holstein (100 y 103,4 cm, respectivamente) (Kienitz et al., 2017). Esta diferencia se puede relacionar a las razas empleadas, ya que el manejo de la alimentación fue diferente para ambos trabajos, diferenciándose en que el presente trabajo se suplementos con alimento comercial (proteína $18 \%$ ).

Las medidas bovinométricas AA, DII y DI, respaldan que las hembras F1 tuvieron un crecimiento y desarrollo más acelerado entre el tercer y séptimo mes, período en el cual los animales se ven sometidos a uno de los cambios más bruscos en función de la alimentación, el destete. Todo lo anterior muestra que a medida que se cuenta con cruzamientos absorbentes basados en aumentar el porcentaje de Kiwi Cross con animales de base Holstein, existe tendencia generacional de disminución de tamaño corporal. Dadas las condiciones de ladera del departamento de Nariño, contar con animales de tamaño mediano puede favorecer a que se tenga menos presión sobre el suelo y contar con una carga animal mayor por área.

\section{Conclusiones}

Los valores obtenidos en el presente estudio mostraron que animales provenientes del cruce entre Kiwi Cross y Holstein se categorizaron por ser de tamaño mediano comparativamente con las razas Holstein y la raza Jersey, que son animales de tamaño grande y pequeño, respectivamente. A medida que se aumentó el porcentaje de sangre Kiwi Cross, se obtuvieron animales de menor tamaño y por ende de menor peso. No obstante, en algunos casos las hembras F1 fueron similares a las de la raza Holstein-Friesian puras y las hembras F2 (75 \% Kiwi Cross y $25 \%$ Holstein) a las de raza Jersey.

Las variables productivas y bovinométricas presentadas en este estudio definieron el crecimiento y tamaño corporal de los animales de reemplazo, lo que podría permitir analizar periódicamente el manejo de los animales y, de esta manera, poder definir el mejor biotipo para cada finca.

En Colombia el uso de Kiwi Cross aún no es tan popular como el Holstein y el Jersey, por lo que se recomienda continuar con evaluaciones que permitan comparar todas las generaciones para determinar su desempeño en condiciones colombianas de trópico alto.

\section{Agradecimientos}

Los autores agradecen el soporte financiero dado por el Ministerio de Agricultura y Desarrollo Rural (MADR) para la ejecución de actividades por la Corporación Colombiana de Investigación Agropecuaria (AGROSAVIA) en el marco del proyecto "Validación del modelo neozelandés en un sistema de producción de leche en Nariño".

\section{Referencias}

Bar-Peled, U., Robinzon, B., Maltz, E., Tagari, H., Folman, Y., Bruckental, I., \& Lehrer, A. R. (1997). Increased weight gain and effects on production parameters of Holstein heifer calves that were allowed to suckle from birth to six weeks of age. Journal of Dairy Science, 80(10), 2523-2528. http://doi.org/10.3168/jds.s0022-0302(97)76205-2

Black, R. A., Whitlock, B. K., \& Krawczel, P.D. (2017). Effect of maternal exercise on calf dry matter intake, weight gain, behavior, and cortisol concentrations at disbudding and weaning. Journal of Dairy Science, 100(9), 7390-7400. http:// doi.org/10.3168/jds.2016-12191 
Boro, P., Naha, B. C., Madkar, A. R., Saikia, D. P, Prakash, C, Godara, A. S., \& Chandrakar, J. (2016). The effects of heifer growth on milk production and efficiency. Internactional Journal of Science and Nature, 7(2), 220-227.

Carulla, J., \& Ortega E. (2016). Sistemas de producción lechera en Colombia: retos y oportunidades. Archivos Latinoamericanos de Producción Animal, 24(2), 83-87.

Departamento Administrativo Nacional de Estadistica. (2020, 30 de junio). Boletín técnico. Encuesta Nacional Agropecuaria (ENA) 2019. https://www.dane.gov.co/

de-Escobar, C., Villalobos, A., \& Núñez, J. (2014). Medidas zoométricas del ganado bovino criolo de Panamá. Investigación y Pensamiento Crítico, 2(5), 26-33.

Hoffman, P. C. (1997). Optimum body size of Holstein replacement heifers. Journal of Animal Science, 75(3), 836-845. http:// doi.org/10.2527/1997.753836x

Hoffman, P. C. (2015). Optimum growth rates for Holstein replacement heifers. University of Wisconsin-Madison. https://fyi. extension.wisc.edu/heifermgmt/files/2015/02/optimumgrowthrates.pdf

Holmann, F., Rivas, L., Carulla, J., Rivera, B., Giraldo, L., Guzmán, S., Martínez, M., Medina, A., \& Farrow, A. (2004). Producción de leche y su relación con los mercados; caso colombiano. Centro Internacional de Agricultura Tropical. http://ciat-library.ciat.cgiar.org/Articulos_Ciat/tropileche/books/Produccion_leche_relacion_mercados_ caso_Colombia.pdf

Holmann, F., Rivas, L., Carulla, J., Rivera, B., Giraldo, L., Guzmán, S., Martínez, M., Medina, A., \& Farrow, A. (2006). Producción de leche y su relación con los mercados; caso colombiano. En: Universidad del Zulia (Ed.), Memorias X Semininario Manejo y utilización de pastos y forrajes en sistemas de producción animal. Producción de leche y su relación con os mercados. (pp. 149-156). Universidad del Zulia.

Khan, M. A., Bach, A., Weary, D. M., \& von Keyserlingk, M. A. G. (2016). Invited review: Transitioning from milk to solid feedin dairy heifers. Journal of Dairy Science, 99(2), 885-902. http://doi.org/ 10.3168/jds .2015-9975

Kienitz, M. J., Heins, B. J., \& Chester-Jones, H. (2017). Growth, behavior, and economics of group-fed dairy calves fed once or twice daily in an organic production system. Journal of Dairy Science, 100(4), 3318-3325. http://doi.org/10.3168/ jds.2016-11885

Kim, Y.H., Toji, N., Kizaki, K., Kushibiki, S., Ichijo, T., \& Sato, S. (2016). Effects of dietary foraje and calf starter on ruminal $\mathrm{pH}$ and transcriptomic adaptation of the rumen epithelium in Holstein calves during the weaning transition. Physiol Genomics, 48(11), 803-809. http://doi.org/10.1152/physiolgenomics.00086.2016

Knight, C., \& Sorenson, A. (2001). Windows in early mammary development: critical or not? Reproduction, 122(3), 337-345. http://doi.org/10.1530/reprod/122.3.337

LIC, \& NZ Dairy. (2016). New Zealand Dairy Statistics 2015-16. https://www.lic.co.nz/about/dairy-statistics/

McCoard, S., Heiser, A., Lowe, K., Molenaar, A., McLean, P., Johnstone, P., Leath, S., Hoskin, S. O., \& Khan, M. A. (2019). Effect of weaning age on growth, mammary gland development, and immune function in Holstein Friesian calves fed conserved alfalfa (FiberStart). Journal of Dairy Science, 102(7), 6076-6087. http://doi.org/10.3168/jds.2018-15615

Macdonald, K. A., Penno, J. W., Bryant, A. M., \& Roche, J. R. (2005). Effect of feeding level pre- and post-puberty and body weight at first calving on growth, milk production, and fertility in grazing dairy cows. Journal of Dairy Science, 88(9), 3363-3375. http://doi.org/10.3168/jds.S0022-0302(05)73020-4 
Macdonald, K., \& Roche, J. (2011). Replacement heifers - rearing the next generation. In NZ Dairy Technical Series (Ed.), Technical Series (pp. 11-13). New Zeland Dairy.

Martínez, R., Fernández, E., Abbiati, N., \& Broccoli, A. (2007). Caracterización zoométrica de bovinos criollos: Patagónicos vs. Noroeste Argentino. Revista MVZ Córdoba, 12(2), 1042-1049.

Mejía, F. 2012. Prospectiva de la cadena láctea del departamento de Nariño al horizonte del año 2020. Tendencias, 13(1), 36-54.

Mogollón, H., Nieto, D., \& Castro, E. (2020). Productive performance of Holstein and the crossbreding Kiwi Cross x Holstein cattle. Agronomía Mesoamericana, 31(2), 329-339. https://doi.org/10.15517/am.v31i2.38541

Monge-Rojas, C. R., \& Elizondo-Salazar, J. A. (2017). Alojamiento individual o en parejas: su efecto sobre el consumo de alimento balanceado y el crecimiento en terneras Jersey. Nutrición Animal Tropical, 11(1), 38-51. http://doi. org/10.15517/nat.v11i1.29171

Montgomerie, W. (2005). Ten years experience of multi-breed evaluations and crossbreeding in New Zealand. In Interbull (Ed.), Proceedings of the 2005 Interbull meeting (pp. 98-101). Interbull.

Mourits, M. C. M., Huirne, R. B. M., Dijkhuizen, A. A., Kristensen, A. R., \& Galligan, D. T. (1999). Economic optimization of dairy heifer management decisions. Agricultural Systems, 61(1), 17-31.http://doi.org/10.1016/s0308-521x(99)00029-3

Rowarth, J. S. (2013). Dairy cows - Economic Production and Environmental Protection. In J. R. Dymond (Ed.), Ecosystem services in New Zealand - conditions and trends (pp. 85-93). Manaaki Whenua Press.

Shamay, A., Werner, D., Moallem, U., Barash, H., \& Bruckental, I. (2005). Effect of nursing management and skeletal size at weaning on puberty, skeletal growth rate, and milk production during first lactation of dairy heifers. Journal of Dairy Science, 88(4), 1460-1469. http://doi.org/10.3168/jds.s0022-0302(05)72814-9

Solarte, C., Rosero, C., Burgos, W., \& Caviedes, I. (2009). Caracterización y evaluación genética de la población bovina lechera del trópico alto de Nariño, para la conformación de núcleos de selección. Universidad de Nariño. https://doi. org/10.13140/2.1.3396.1762

Van-Amburgh, M. E., Galton, D. M., Bauman, D. E., Everett, R. W., Fox, D. G., Chase, L. E., \& Erb, H. N. (1998). Effects of Three Prepubertal Body Growth Rates on Performance of Holstein Heifers During First Lactation. Journal of Dairy Science, 81(2), 527-538. http://doi.org/10.3168/jds.s0022-0302(98)75604-8

Vargas-Ramírez, A. M., \& Elizondo-Salazar, J. A. (2014). Determinación de consumo de alimento balanceado y agua, y medidas de crecimiento en terneras Holstein en una finca lechera comercial. Nutrición Animal Tropical, 8(2), 36-50. http:// repositorio.ucr.ac.cr/bitstream/handle/10669/13544/17253-35290-1-SM.pdf?sequence=1\&isAllowed=y

Wattiaux, M. (2011). Heifer raising - birth to weaning. In M. Wattiaux (Ed.), Dairy essentials-raising dairy heifers (Chapter 35, pp. 137-140). Badcock Institute for International Dairy Reseach and Development, University of Wisconsin. http:// www.infodairy.com/infodairy_upload_files/Cows_heifers_calves/Heifers/0192Measuring\%20of\%20growth\%20 level-e.pdf

World Holstein-Friesian Federation. (2005). Evaluación morfológica internacional del vacuno de leche. http://www.euholsteins. com/info/typetraits/type_esp_2005-2.pdf

Zanton, G. I., \& Heinrichs, A. J. (2005). Meta-Analysis to Assess Effect of Prepubertal Average Daily Gain of Holstein Heifers on First-Lactation Production. Journal of Dairy Science, 88(11), 3860-3867. http://doi.org/10.3168/jds.s00220302(05)73071-X 